\title{
Differential Labelling of the Vegetative Primary Nucleus of the Marine Green Alga Acetabularia by Lectins, Anti- immunoglobulin-antibodies and Antinuclear Autoimmunosera
}

\author{
G. Tischendorf, D. Sawitzky and G. Werz \\ Division of Biology, Department of Ultrastructure Research and Electron \\ Microscopy, Freie Universität Berlin, Königin-Luise-Str. 12-16a, \\ D-1000, Berlin 33, Germany
}

Accepted September 9, 1986

The highly conservative ultrastructural morphology of nuclei in both unicellular and multicellular organisms gives rise to a general expansion of models for subnuclear assembling structures and their molecular functions originally valid for a single type of cell nucleus. Structural conservatism can be studied by the comparative analysis of the individual components forming the complex subnuclear structural entities. A structural homology is evident, for example, if immunoglobulins or lectins specific for a nuclear antigen of one species cross-react with individual components of nuclei from diverse sources.

Using antinuclear autoimmuno-antibodies of human origin, as well as some lectins and anti-immunoglobulin-antibodies (AIAs), for comparative analysis of nuclei by immunofluorescence microscopy we have determined structural homologies between nuclear components that belong to extremely diverse eukaryonts, namely, vertebrates and the unicellular marine green alga Acetabularia.

\section{Materials and methods}

The experiments were performed with isolated nuclei of the unicellular and uninucleate marine green alga Acetabularia. The algae were grown in Schreiber medium according to Hämmerling (1944).

After dipping a separated rhizoid in one of the prefixation agents (see below), the primary nucleus was squeezed out of the rhizoid. The isolated nucleus was immediately transferred to a glass slide coated with gelatine, or to an uncoated one, and was flattened by a siliconecoated cover slide. After freezing in $\mathrm{LN}_{2}$ the cover glass was removed and the nucleus was postfixed in one of the postfixation agents (see below), each containing $3.5 \%$ formaldehyde.

The flattened nucleus remained fixed to the glass support during all subsequent incubation and washing procedures, in contrast to non-flattened ones. However, without prefixation the nuclear structure was destroyed by the pressure of the cover slide. Nuclei, prefixed for 2 min and flattened in various salt environments, i.e., in "high salt" (100 mM PBS, pH 7.2), "intermediate salt" (50 mM PBS, pH 7.2), or "low salt" (10 mM PBS, pH 7.2), showed minimal morphological differences when analyzed in light microscopical phase contrast. Thus, the prefixation agent was structure conserving within a wide range of experimental conditions.

Prefixation: The isolated nuclei were prefixed for $2 \mathrm{~min}$ in FA-PBS-A1 $(1.5 \%$ formaldehyde in W-PBS-1 (see below)), FA-PBS-A2 (1.5\% formaldehyde in $100 \mathrm{mM} \mathrm{Na-K-phosphate}$ buffer, $0.9 \% \mathrm{NaCl}, 5 \%$ sucrose; $\mathrm{pH} 7.2)$, or FA-PBS-A3 (1.5\% formaldehyde, $5 \%$ sucrose in W-PBS-3 (see below)).

Postfixation: The nuclei were postfixed for $2 \mathrm{~min}$ in FA-PBS-B1 $(3.5 \%$ formaldehyde in W-PBS-1 (see below)), FA-PBS-B2 (3.5\% formaldehyde in $100 \mathrm{mM} \mathrm{Na-K-phosphate} \mathrm{buffer,}$ 
$0.9 \% \mathrm{NaCl} ; \mathrm{pH} 7.2$ ), or in FA-PBS-B3 (3.5\% formaldehyde in W-PBS-3 (see below)).

Washing: After postfixation, nuclei were immediately washed in phosphate buffered saline: W-PBS-1 (20 mM Na-K-phosphate buffer, $0.9 \% \mathrm{NaCl} ; \mathrm{pH} 7.4)$, W-PBS-2 (1.5 mM/L

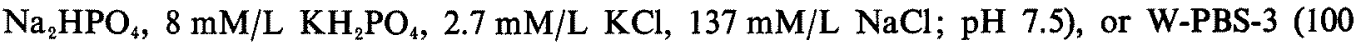
$\mathrm{mM} \mathrm{Na-K}$-phosphate buffer, $50 \mathrm{mM} / \mathrm{L} \mathrm{KCl}, 0.9 \% \mathrm{NaCl} ; \mathrm{pH} 7.2)$. Thereafter, all but a thin film of washing solution was withdrawn.

Labelling of nuclei: After washing, the nuclei were incubated for $30 \mathrm{~min}$ with antibody/ lectin solution (see legends to figures for details).

Non-fluorescent immunoglobulins as primary markers: ANA-H (anti-nuclear antibodies from human serum; homogeneous reaction pattern with human/vertebrate nuclei; Sigma, St. Louis, USA), ANA-N (anti-nuclear antibodies from human serum; nucleolar reaction pattern with human/vertebrate nuclei; Sigma, St. Louis, USA), ANA-S (anti-nuclear antibodies from human serum; speckled reaction pattern with human/vertebrate nuclei; Sigma, St. Louis, USA), LED (serum from patient with lupus erythematosus disseminatus), or CEA (human serum with carcino-embryonic antigen $(2.660 \mu \mathrm{g} / \mathrm{ml}))$ were used as primary markers for indirect immunostaining of the algal nuclei.

Fluorescent immunoglobulins as primary or secondary markers: Sp-anti-man-FITC (antihuman immunoglobulins from sheep, conjugated with fluorescein-isothiocyanate; Wellcome, UK), gt-anti-man-RB-200 SC (anti-human immunoglobulins from goat, conjugated with lissamine rhodamine B 200 sulphonyl chloride; Medac, FRG), gt-anti-man-RITC (anti-man IgG from goat, conjugated with rhodamine-isothiocyanate; Miles Laboratories, USA), and rbanti-ms IgG-FITC (anti-mouse IgG from rabbit; conjugated with fluorescein-isothiocyanate; Miles Laboratories, USA) were used for direct and indirect immuno-staining of the algal nucleus.

Lectins conjugated with FITC: WGA-FITC (wheat germ agglutinin; Medac, FRG), UEA-I-FITC (Ulex europeus I; Medac, FRG), or GS-I-FITC (Griffonia simplicifolia isolectin B4; Medac, FRG) were used for direct staining of the algal nuclei.

After final washing (see legends to figures), the nuclei were examined in a Leitz Ortholux II microscope equipped with phase contrast and epifluorescence devices. Filter combinations I2 (BP 450-490/RKP 510/LP 515) for FITC-fluorochrome and N2 (BP 350-560/RKP 580/LP 580) for RITC or RB 200-SC fluorochromes were used. Pictures were taken on Ilford HP5 films.

\section{Results}

Human autoimmunosera cross-react with the algal nucleus

Subtypes $\mathrm{H}, \mathrm{N}$ and $\mathrm{S}$ of human autoimmunosera with antinuclear specificity (ANA) and LED or CEA each gave a cross-reaction with the vegetative nucleus of the green alga Acetabularia. They formed a homogeneous (ANA-H; Figs. 1e-f), nucleolar (ANA-N; Figs. 1a-b), speckled (ANA-S; Figs. 1g-h), or perinucleolar (LED and CEA; Figs. 2a-b and c-d, respectively) pattern of reaction when using the technique of indirect immunoflorescence in combination with AIA-FITC. In the absence of primary markers, the fluorescent AIAs did not bind to the nucleus (Figs. 2e-f).

The speckled, homogeneous, and nucleolar patterns (Figs. 1a-b, e-f, and g-h, respectively) resembled those formed by the three ANA-subtypes with vertebrate or human nuclei. They are the most prominent patterns observed in the latter (for review, see Seelig et al. 1983). Crossreaction and pattern homology demonstrate that Acetabularia and vertebrate/human nuclei share extremely conservative antigenic determinants.

The corresponding antigens in vertebrate nuclei are 4-6S RNA, 7S-RNA, U3-RNP and 
other nucleolar components (nucleolar pattern), nonhistone proteins or RNP (U1-RNP, Sm, SS-B, Scl-70, PM-1, Jo-1, Ku, Mi-1, PCNA, and centromeres) (speckled pattern), or ds-DNA, ss-DNA and histones (homogeneous pattern; Seelig et al. 1983).

Antibodies of both the LED and CEA types of human serum produced a perinucleolar cross-reaction in Acetabularia (Figs. 2a-d). The antigens recognized by LED might be dsDNA, DNP, U1-RNP, Sm, SB, and nucleolar ones like those in vertebrates (Seelig et al. 1983, Lerner and Steitz 1981, Busch et al. 1982). In the case of CEA, antibodies specific for incre-
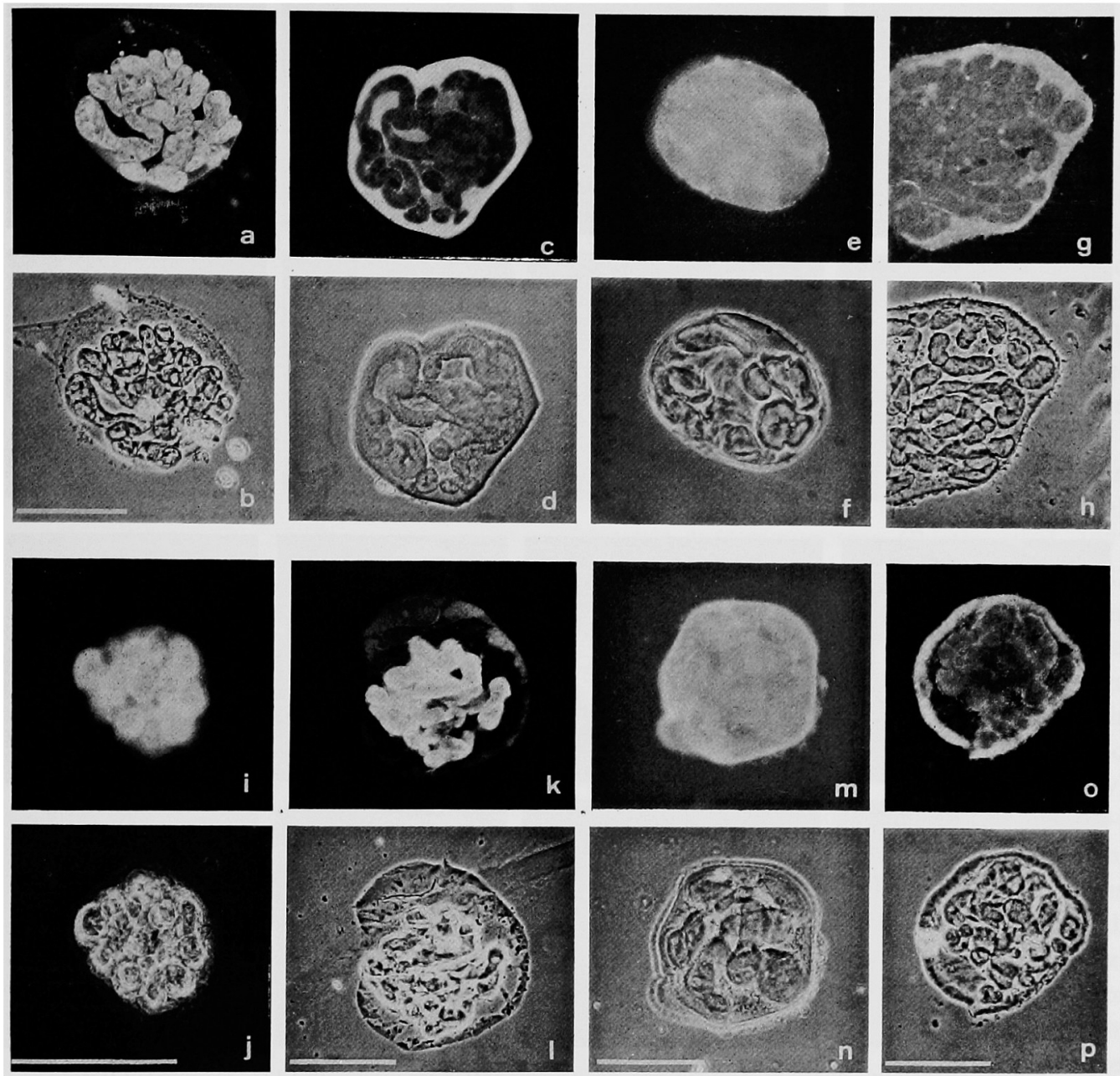

Fig. 1. Vegetative nuclei from Acetabularia mediterranea after reaction with FITC-conjugated leectins (c-d, i-l), with AIA-FITC (m-p), or with AIA/AIA-FITC (a-b, e-h). Nuclei were prefixed in FA-PBS-Al (i-l, o-p), FA-PBS-A2 (a-b), or FA-PBS-A3 (c-d, g-h, m-n) on glass slides coated with $1 \%$ gelatine $(i-1,0-p)$ or uncoated $(a-h, m-n)$. After postfixation in FA-PBS-B1 (i-l, o-p), FA-PBS-B2 (a-b), or FA-PBS-B3 (c-h, m-n), they were washed in W-PBS-1 (i-1, o-p), W-PBS-2 (a-b), or W-PBS-3 (c-h, m-n). The nuclei shown in $i-l$ and o-p were brought to reaction with antibodies/lectins immediately after they had dried onto the glass slides. a-b, ANA-N 1: 10/spanti-man-FITC 1:10. c-d, WGA-FITC, undiluted. e-f, ANA-H 1:10/sp-anti-man-FITC 1: 10. g-h, ANA-S 1: 10/sp-anti-man-FITC $1: 10$. i-j, GSI-FITC, undiluted. k-1, UEA-I-FITC, undiluted. $\mathrm{m}-\mathrm{n}$, anti-man-RB-2000 SC, undiluted. o-p, rb-anti-ms-FITC, undiluted. Markers were diluted with W-PBS-2 (a-b: "low salt" conditions) or with W-PBS-3 (e-h: "high salt" conditions). (For solutions, see Materials and Methods.) The bar represents $100 \mu \mathrm{m}$. 
asingly produced fetal antigens of proliferating cells might be involved (Busch et al. 1983). Lectins and AIAs react with the algal nucleus

WGA, UEA and GS-I and, unexpectedly, antibodies from animals, hyperimmune against immunoglobulins, also reacted with the Acetabularia nucleus. Again the antigens recognized were differently distributed. They were part of the nucleoli (Figs. 1i-1, 2k-1), caryoplasm (Figs. 1c-d, $m-n$ ), and nuclear envelope (Figs. $1 \mathrm{~m}-\mathrm{p}$ ). The findings point to glycosidic nu-
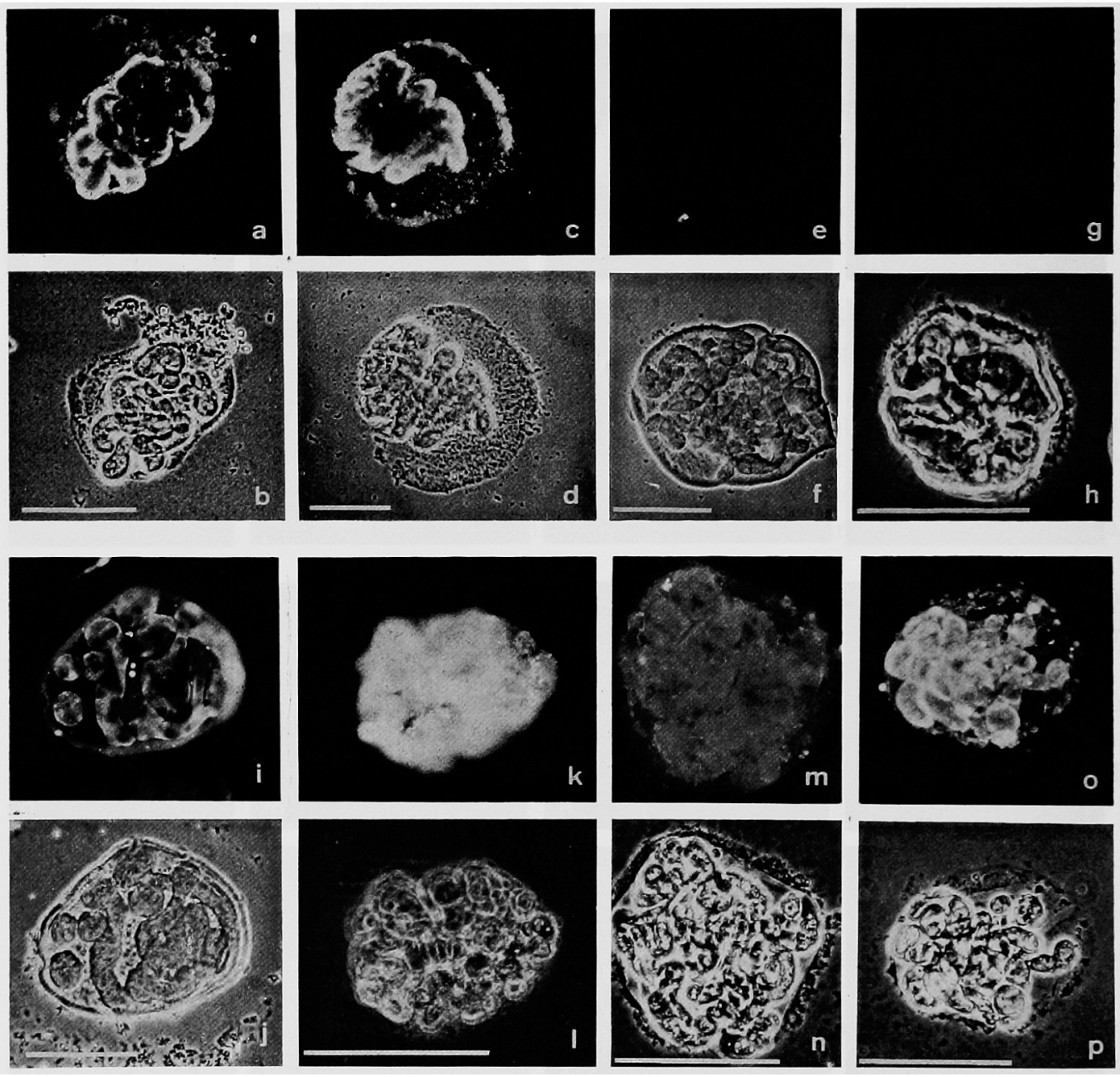

Fig. 2. Vegetative nuclei of Acetabularia mediterranea after reaction with FITC-conjugated lectins (i-1), AIA-FITC (e-h), or AIA/AIA-FITC (a-d, m-p). Nuclei were prefixed in FA-PBS-A1 (a-d, g-h, k-l), FA-PBS-A2 (m-p), or FA-PBS-A3 (e-f, i-j, m-n) on glass slides coated with $1 \%$ gelatine (a-d, g-h, k-l) or uncoated (e-f, i-j, m-p). After postfixation in FA-PBS-B1 (a-d, g-h, k-l), FAPBS-B2 (m-p), or FA-PBS-B3 (e-f, i-j), they were washed in W-PBS-1 (a-d, g-h, k-l), W-PBS-2 $(\mathrm{m}-\mathrm{p})$, or W-PBS-3 (e-f, $\mathrm{i}-\mathrm{j})$. The nuclei in $\mathrm{a}-\mathrm{d}, \mathrm{g}-\mathrm{h}$ and $\mathrm{k}-\mathrm{l}$ were brought to reaction with antibodies/lectins immediately after they had dried onto the glass slides. a-b, LED 1:10/sp-antiman-FITC 1:10. c-d, CEA 1: 10/sp-anti-man-FITC 1:10. e-f, sp-anti-man-FITC 1: 10 (control). g-h, gt-anti-man-RITC, undiluted. i-j, ANA-N 1:10/sp-anti-man-FITC 1:10. k-l, WGA-FITC, undiluted. m-n, ANA-H 1:10/sp-anti-man-FITC 1:10. o-p, ANA-S 1:10/spanti-man-FITC 1: 10. Dilution of markers in a-d was with W-PBS-1 and in $m-p$ with W-PBS-2 ("low salt" conditions). In e-f and $\mathrm{i}-\mathrm{j}$ markers were diluted with W-PBS-3 ("high salt" conditions).

(For solutions, see Materials and Methods.) The bar represents $100 \mu \mathrm{m}$. 
clear determinants at the sites of reaction. Among these must be terminal and internal $\alpha$-DGlc-NAc (core in Asp-linked glyco-chains in combination with terminal or internal sialic acid; reactive with the $34 \mathrm{Kd} \mathrm{WGA}$ ), $\alpha$-L-fucose, and, thus, blood group O substance (reactive with the $170 \mathrm{Kd}$ UEA-I), and $\alpha$-D-Gal, the blood group B substance (reactive with the $114 \mathrm{Kd}$ GS-I) (see Lis and Sharon 1977 for review). In the case of cross-reacting AIA, most probably those antibodies specific for the glycosidic part of the antibody molecule bound to the algal nucleus. This can be deduced from the fact that vertebrates are the only source of immunoglobulins.

\section{Discussion}

The vegetative primary nucleus of Acetabularia is unusually large (diameter of up to 150 $\mu \mathrm{m}$ or more) and reveals a very high transcriptional activity. In addition to an abundance of pore complexes (about 90 per $\mu \mathrm{m}^{2}$; Zerban and Werz 1975a, b), it possesses other structures of interest in extraordinarily large numbers. For example, about 10 "macronucleoli", each with a diameter of up to $10 \mu \mathrm{m}$, are the rule. A transcriptionally highly active nucleus of extraordinary size, but conservative morphology, like that of Acetabularia, could be expected to be a depot for one or another conservative nuclear antigen. Therefore, it is possibly a key object to the identification, isolation and characterization especially of those conservative antigens which appear in only extremely small quantities in nuclei from other sources.

Antinuclear antibodies are ideal tools to detect determinants common to nuclear antigens in different species because of their potential cross-reaction. A natural reservoir of selectively binding marker molecules already exists. These are autoimmunosera (Bustin et al. 1977, Silver and Elgin 1978, Lerner and Steitz 1979, 1981, Kurth and Bustin 1981, Busch et al. 1982, 1983, Stetler et al. 1982, McKeon et al. 1983, Seelig et al. 1983) and lectins (Virtanen and Wartiovaara 1976, Rizzo and Bustin 1977, Kurth et al. 1979, Hozier and Furcht 1980, Ben-Ze'ev and Abulavia 1983), some of which have been tested here for cross-reaction with the green algal nucleus. The distinct reaction patterns each appear under unique preparative conditions. The nucleolar pattern requires relatively "low salt" (Figs. 1a-b). The speckled as well as the homogeneous patterns appear only under "high salt" conditions (Figs. $1 \mathrm{~g}-\mathrm{h}$, e-f, respectively). Otherwise, the reaction patterns are different from the ones described (see Figs. $2 i-j, m-n, o-p$ for ANA-N, ANA-H and ANA-S, respectively). It is suggested that this is caused by masking of determinants, migration of antigens, or accessibility to other, originally masked antigens. Therefore, "new" antigens could bind "new" antibodies. Those "new" antibodies already exist among the polyspecific test sera. Thus, serum of the "speckeld type" (Figs. 1g-h) may appear nucleolar specific (Figs. 20-p) under certain preparative conditions when it, in addition to the "speckled type" of antibodies, also contains those of the anti-nucleolar type. In the same way, the anti-nucleolar serum (Figs. 1a-b) simultaneously adopts the homogeneous reaction (Figs. 2i-j), whereas the serum of the "homogeneous type" (Figs. le-f) shows faint nucleolar reaction (Figs. $2 \mathrm{~m}-\mathrm{n}$ ). In addition to this, the concentrations of labelling-immunoglobulins usually differ. In lower titer ranges a nucleolar pattern might change to a speckeld one, and the latter again to a homogeneous pattern. Although not yet proven, we assume that valence, affinity and antibody concentrations are prominent factors.

Coating the glass slides with $1 \%$ gelatine (Figs. 1i-1, o-p, and Figs. 2a-d, g-h, k-1) or using them uncoated (other Figures) also influences the quality of immunofluorescence. Potential acidity of unbuffered gelatine may locally affect the contiguous nuclear structures. Local effects may also arise from the high salt content of neutralized gelatine after it has dried onto the slide. For example, nuclei which have been air-dried on glass slides coated with gelatine cannot be stained by a gt-anti-man-RITC "low salt" overlay (Figs. $2 \mathrm{~g}-\mathrm{h}$ ). The AIA, however, stains 
the nucleoli and envelope of nondried nuclei on uncoated glass slides.

Some of the conservative determinants detected in the primary nucleus of Acetabularia are glycosidic. Glycosidic determinants obviously are a general part of the nuclear reserve. They are found in conjunction with nonhistone chromosomal proteins of the detergent-resistant nuclear matrix (nuclear frame work) or with proteins of the easily extractable chromatin, as well as with the nuclear pore lamina complex (Silver et al. 1978, Zegarelli-Schmidt et al. 1980, Kurth and Bustin 1981).

\section{Acknowledgements}

The authors wish to thank Ms. E. Nielsen for assistance in the preparation and typing of the manuscript. This research was supported in part by a grant from the Deutsche Forschungsgemeinschaft (Ti 136/1-2).

\section{References}

Ben-Ze'ev, B. and Abulavia, R. 1983. Association of glycoconjugates with the cytoskeletal framework. Molec. and Cell. Biol. 3: 684-692.

Busch, H., Busch, R. K. and Chan, P.-K. 1983. Tumor nucleolar antigens. In: Chromsomal Nonhistone Proteins (L. S. Hnilica, Ed.), Vol. 2: Immunology, pp. 58-103. CRC Press Inc., Boca Raton, Florida.

-, Reddy, R., Rothblum, L. and Choi, Y. C. 1982. SnRNAs, snRNPs, and RNA processing. Ann. Rev. Biochem. 51: 617-654.

Bustin, M., Kurth, P. D., Moudrianakis, E. N., Goldblatt, D., Sperling, R. and Rizzo, W. B. 1977. Immunological probes for chromatin structure. Cold Spring Harbor Symp. Quant. Biol. 42: 379-388.

Hämmerling, J. 1944. Zur Lebensweise, Fortpflanzung und Entwicklung verschiedener Dasycladaceen. Arch. Protistenkunde 87: 7-56.

Hozier, J. and Furcht, L. T. 1980. Binding of lectins to mitotic chromosomes and interphase nuclear substructures. Cell Biol. Intern. Rep. 4: 1091-1099.

Kurth, P. D. and Bustin, M. 1981. Localization of chromosomal protein HMG-1 in polytene chromosome of Chironomus thummi. J. Cell Biol. 89: 70-77.

-, - - and Moudrianakis, E. N. 1979. Con A binds to puffs in polytene chromosome. Nature 779: 448-450.

Lerner, M. R. and Steitz, J. A. 1979. Antibodies to small nuclear RNAs complexed with proteins are produced by patients with systemic lupus erythematosus. Proc. Natl. Acad. Sci. USA 76: 5495-5499.

- and - 1981. Snurps and scyrps. Cell 25: 298-300.

Lis, H. and Sharon, N. 1977. Lectins: Their chemistry and application to immunology. In: The Antigens (M. Sela, Ed.), Vol. IV, pp. 429-529. Academic Press, New York.

McKeon, F. D., Tuffanelli, D. L., Fukuyama, K. and Kirschner, M. W. 1983. Autoimmune response directed against conserved determinants of nuclear envelope proteins in a patient with linear scleroderma. Proc. Natl. Acad. Sci. USA 80 : 4374-4378.

Rizzo, W. B. and Bustin, M. 1977. Lectins as probes of chromatin structure. J. Biol. Chem. 252: 7062-7067.

Seelig, H. P., Liehr, H. and Seelig, R. 1983. Antikörper gegen Zellkernantigene. G. Fischer, Stuttgart.

Silver, L. M. and Elgin, S. C. R. 1978. Production and characterization of antisera against three individual NHC-proteins: a case of a generally distributed NHC-protein. Chromosoma 68: 101-114.

-, Wu, C. E. C. and Elgin, S. C. R. 1978. Immunofluorescence techniques in the analysis of chromosomal proteins. Methods in Cell Biology 18: 151-231.

Stetler, D. A., Rose, K. M., Wenger, M. E., Berlin, C. M. and Jacob, S. T. 1982. Antibodies to distinct polypeptides of RNA polymerase $I$ in sera from patients with rheumatic autoimmune disease. Proc. Natl. Acad. Sci. USA 79: 7499-7503.

Virtanen, I. and Wartiovaara, J. 1976. Lectin receptor sites on rat liver cell nuclear membranes. J. Cell Sci. 22: 335-344.

Zegarelli-Schmidt, E., Silver, E. M., Eastman, E. and Goodman, R. M. $1980 . \quad$ Conservation of nonhistone chromosomal proteins through Diptera evolution. Chromosoma 79: 65-74.

Zerban, H. and Werz, G. 1975a. Zahl und Verteilung von Kernporenkomplexen bei Acetabularia in Abhängigkeit verschiedener Entwicklungsstadien. Beitr. Elektronemikr. Direktabb. Oberf. 8: 709-727.

- and - 1975b. Changes in frequency and total number of nuclear pores in the life cycle of Acetabularia. Exp. Cell Res. 93: 472-477. 